\title{
Using waves to generate electricity
}

\section{Anne Blavette}

\author{
Hydraulics and Maritime Research Centre, UCC
}

\section{Introduction}

In less than a century, electricity has become the cornerstone of our modern society. Thanks to it, living conditions have changed more dramatically in 50 years than in two centuries. However, whereas electricity has provided well-being to billions of people over these past few decades, its ever-growing consumption is more and more threatening us. Climate change, fossil fuel depletion, and security of energy supply are crucial issues to be addressed for our "energy society" to be sustainable. Renewables are considered as a major part of the solution. This article deals with the issues related to integrating wave energy into electrical power systems.

\section{Wave energy grid integration}

The major problem associated with wave energy is its variability. Variability can be observed at several time scales: from the range of seconds (wave-to-wave), to minutes (seastate to sea-state), to days or months (seasonal variations).

However, if wave energy is to be used for generating electricity, electrical networks must be operated so as to cope with this inherent variability. If this is not the case, the effects on electrical grids may range from bulb flicker to total black-outs, which is clearly unacceptable.

The most efficient way to obtain a better understanding of the potential effects of wave electricity on networks is to perform dynamic simulations. These simulations involve a numerical electrical network simulator and generation plant numerical models. The network simulator enables its user to simulate any electrical network (at either a local or a national/international level). The network modelled in this simulator reacts as the real electrical grid would to any disturbance applied to it. Numerical models are implemented in the simulator to represent any power plant, either fuel-fired, hydro-driven or wind farms. However, there is as yet no model available for so-called "wave farms" yet. This lack of models prevents electrical system operators from studying the potential effects of wave electricity on their networks. Consequently, no wave farm is allowed to connect to the grid, apart from small test devices. This represents a major barrier for the development of the wave energy industry on a massive scale and the International Energy Agency has hence included generic modelling as one of its key research topics. 


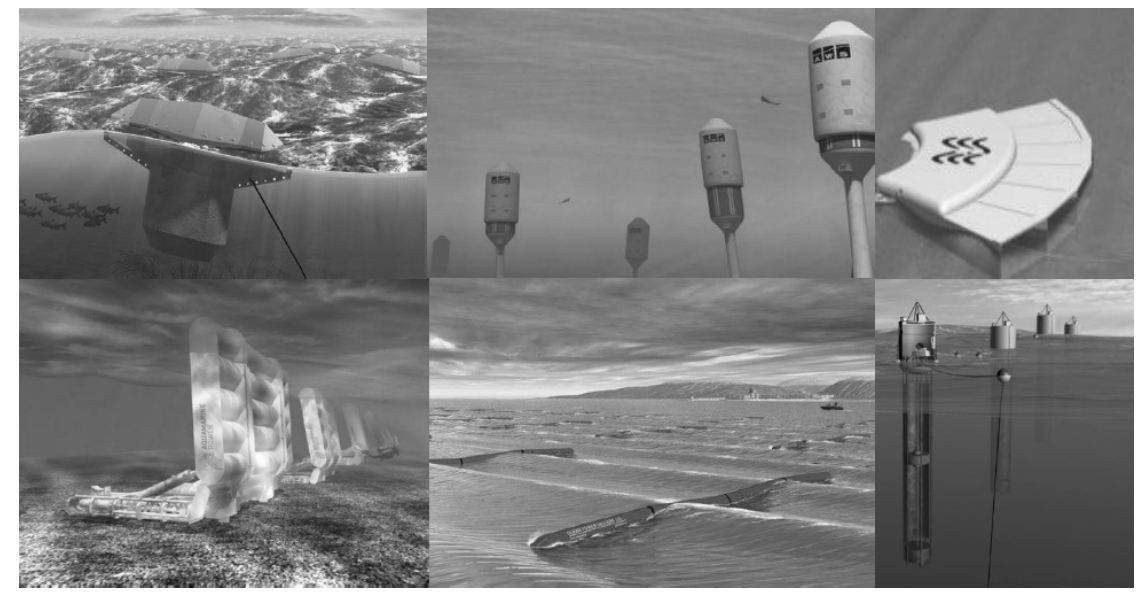

Figure 1: Some device technologies

\section{Modelling of wave energy converters}

The modelling of wave farms and of its individual devices (called wave energy converters) is still in its infancy stage as it is a new field of research. For the purpose of my work, two modelling approaches were considered.

\section{Technology-specific modelling}

The first approach consists in modelling device technologies specifically but was eventually deemed as irrelevant. There are actually a myriad of wave converter technologies (see Figure 1). As of now (July 2010), I have listed almost 170 different devices, none of which can be clearly identified as the leading technology. Besides, modelling entirely and precisely every device requires much time as the device technologies are fundamentally different.

\section{Generic modelling}

The second approach intends to be much more all-encompassing: it is called "generic modelling". My main topic of research, along with the influence of marine electricity on the grid, is generic modelling. In the course of my $\mathrm{PhD}$ work, I intend to create a theoretical generic model and then implement it as a numerical model in a network simulator. From this, I will be able to study the influence of marine electricity on the network as realistically as possible.

A generic model is a model whose structure is constant, but whose internal parameters can be tuned for each device technology. As a result, it is hoped that many devices (as much as possible) could be modelled by means of one single generic model. If an example 
is to be given, it may be the one of a train: the structure of the train (its coaches and locomotive) does not change but its passengers do; each journey is hence different.

This model will, of course, have to be as precise as possible and it is intended to check its output against field data obtained from wave energy converters tested at sea or in a wave basin. The first step towards a generic model is the collection of common features among all devices. Each device is currently being fully documented and a general outline classification being extracted.

A generic model will be a major breakthrough and will enable the wave energy industry to avoid some pitfalls encountered by the wind energy industry. Wind turbine developers faced the same problem several years ago as they had to provide numerical models of their machines to electrical grid operators. One of the major issues at this time was that they had little knowledge of electrical network engineering and often subcontracted the modelling to third parties (most often to universities). This situation was very sensitive with respect to commercial confidentiality. Besides, the models were not created at the same level of detail (and hence not for the same time step of simulation): this discrepancy in modelling detail led to implementation difficulties because of undesired interactions between the models. As of now, although the main wind turbine developers have been involved in numerical modelling for years, no realistic wind turbine generic model has been issued yet, but only specific ones. However, it should be stressed (as a matter of comparison) that whereas almost all wind turbines have a similar 3-bladed structure, marine converter technologies (as mentioned earlier) are much more eclectic, increasing the complexity of their modelling.

\section{Conclusion}

To conclude, this paper details how powerful a generic model will be and what a major breakthrough it will represent for the wave energy industry and for the electrical system operators. More generally, this article stresses the necessity of studying the impact of wave electricity on electrical networks. It is hoped as well that this paper illustrates the important opportunities for creativity and innovation that exist in wave energy research.

Anne Blavette is a student at the Hydraulics and Maritime Research Centre (UCC) under the supervision of Dara O'Sullivan, Michael Egan, Hydraulics and Maritime Research Centre UCC and Tony Lewis, Electrical Engineering Department UCC. The author would like to acknowledge them for their always most appreciated help and the Charles Parsons Initiative for funding her research. 